\title{
Diagnostic Performance of SPECT/CT Versus Diffusion-Weighted Mri in Characterization of Indeterminate Osseous Lesions Detected on Bone Scan
}

\section{Maha Khalil}

South Egypt Cancer Institute

Yasser Gaber Abdelhafez ( $\nabla$ yga1979@gmail.com )

South Egypt Cancer Institute https://orcid.org/0000-0002-1989-3094

Ahmed A. Kandeel

Cairo University Kasr Alainy Faculty of Medicine

\section{Gehan S Seifeldein}

Assiut University Faculty of Medicine

Haisam Atta

South Egypt Cancer Institute

\section{Research Article}

Keywords: Single-Photon Emission-Computed Tomography, Diffusion-weighted imaging, MRI, indeterminate solitary osseous lesion, bone metastasis, bone scan

Posted Date: February 22nd, 2021

DOI: https://doi.org/10.21203/rs.3.rs-202039/v1

License: (c) (1) This work is licensed under a Creative Commons Attribution 4.0 International License.

Read Full License 


\section{Abstract}

Background: Characterization of solitary osseous lesions on planar bone scintigraphy (PBS) is challenging. In this pilot study, we aimed to explore the diagnostic performance of ${ }^{99 \mathrm{~m} T c-M D P}$ bone scintigraphy using SPECT/CT in comparison to magnetic resonance imaging including diffusionweighted imaging (MR-DWI) in patients with solitary indeterminate osseous lesions detected on PBS.

Methods: This pilot study prospectively recruited 46 cancer patients who underwent PBS, with a finding of a solitary osseous lesion that was deemed indeterminate by two nuclear medicine physicians not involved in subsequent reading. A targeted SPECT/CT and MR-DWI were read independently by two nuclear medicine physicians and two radiologists, respectively, on a 5-point probability score. The final diagnosis of disease status was formulated from subsequent clinical/imaging follow-up within six months.

Results: Agreement between SPECT/CT readers was 0.73 (95\% confidence interval [Cl]:0.51-0.85) and 0.99 (95\%Cl:0.99-1.00) between MR-DWI readers.

According to the follow-up, $16 / 46$ patients proved to have osseous metastasis. The area under the curve (AUC) from ROC analysis was 0.79 (95\% Cl:0.65-93) for SPECT/CT and 0.73 (95\% Cl:0.57-0.89) for MRDWI. No statistically significant difference was noted regarding sensitivity or specificity. MR-DWI was more accurate for lesions located in the appendicular skeleton $(n=17 ; A U C=0.92,95 \% \mathrm{Cl}: 0.68-1.0)$ compared to axial lesions ( $n=29 ; A U C=0.65,95 \% \mathrm{Cl}: 0.45-0.81$ ). The performance of SPECT/CT was comparable for both sites. Diagnostic indices from both modalities were comparable for sclerotic and non-sclerotic lesions on the CT part of SPECT/CT.

Conclusions: In this exploratory report, no significant difference was seen in the overall diagnostic performance between SPECT/CT and MR-DWI for characterization of solitary indeterminate osseous lesions depicted on PBS. MR-DWI results were more reproducible; however, the overall accuracy of MRDWI varied according to lesion site, density patterns on $\mathrm{CT}$, and the primary tumor type. Such differences were less obvious with SPECT/CT.

\section{Introduction}

Bone is the third most common site of cancer metastases. The involvement of the skeleton mostly changes the tumor staging, the overall prognosis, and could radically alter the management plan [1].

Imaging is the cornerstone for detecting bone involvement in cancer patients. Planar bone scintigraphy (PBS) using diphosphonate compounds is the most commonly used modality to survey the whole skeleton for metastases [2]. Although PBS scanning is a sensitive tool, it frequently lacks specificity, and inconclusive reporting is fairly common, especially when a solitary osseous lesion is depicted [3]. Many benign etiologies can cause this finding; examples include degenerative, arthritic, or post-traumatic 
changes. The diagnostic algorithm of such lesions frequently involves follow-up bone scanning or targeted cross-sectional anatomical imaging (CT and/or MRI) to resolve the underlying pathology [4].

The introduction of hybrid SPECT/CT machines integrates anatomical mapping and morphological characterization with the functional tracer distribution in one study. Skeletal SPECT/CT has been shown to overcome many of the limitations encountered with PBS leading to superior diagnostic output [5-11]. However, the uptake of phosphonate bone-seeking tracers reflects only the sites of osteoblastic activity and bone formation [12]; accordingly, it does not adequately accumulate in early bone marrow deposits, which typically do not provoke a secondary osseous bone response. Furthermore, SPECT/CT machines are not widely available, and the procedure could be very expensive.

MRI is known to be more sensitive than CT or radionuclide bone scans for detecting early marrow-based metastases through its superior contrast and better spatial resolution $[13,14]$. On the other hand, the classic morphological MR sequences may fail to accurately differentiate malignant from benign lesions as the signal characterization may overlap. The emergence of functional imaging, together with the advances in MR hardware and software, lead to improved visualization and quantification of the diffusion of water molecules across biological membranes and diffusion-weighted (DW) MRI became more readily available sequence for assessment of bone marrow lesions [1-3]. The assumption is that malignant soft tissue lesions with hypercellularity and reduced extra-cellular matrix would display restricted mobility of water molecules and lower mean apparent diffusion coefficient (ADC, calculated in units of $\mathrm{mm}^{2} / \mathrm{sec}$ ) [15-20]. Bone marrow is peculiar in many aspects, and the malignant marrow lesions show high $A D C$ values than the normal bone marrow [6].

In this prospective study, we evaluated the diagnostic performance of ${ }^{99 \mathrm{~m}} \mathrm{Tc}-\mathrm{MDP}$ bone scintigraphy using SPECT/CT in comparison to MRI, including diffusion-weighted imaging (MR-DWI), in patients with solitary indeterminate osseous lesions detected on planar bone scintigraphy (PBS).

\section{Methods}

\section{Participants}

The Institutional Review Board approved this prospective study. During the period from November 2014 to February 2016, consecutive patients with a known primary tumor, referred to perform planar bone scintigraphy (for staging or re-staging/follow-up), were considered eligible for this study if their PBS showed a solitary osseous lesion, which was deemed "indeterminate" based on PBS by consensus reading of two physicians not involved in this research study. Accordingly, the patient underwent a targeted SPECT/CT acquisition on the same day and MR-DWI within one month from the date of bone scanning.

We excluded patients with anterior rib lesions because the respiratory movement, which is more appreciated anteriorly, could degrade MR-DWI acquisitions [21]. Patients who cannot perform or have 
contraindications for MRI examination, as those having claustrophobia, cardiac pacemaker, or metallic prosthesis not compatible with MRI, were also excluded.

\section{Planar bone scintigraphy and SPECT/CT imaging}

PBS was performed using a hybrid SPETC/CT machine (Symbia T, Siemens Medical Solutions, Erlangen, Germany), 2-4 hours after IV injection of 20-25 mCi (740-925 MBq) 99m-technetium methylene diphosphonate ( $\left.{ }^{99 \mathrm{~m}} \mathrm{Tc}-\mathrm{MDP}\right)$. The dose was modified according to Webster's rule for pediatric patients [22]. The patients were asked to lie supine with arms down. Anterior and posterior projection images of the whole skeleton were acquired using a parallel-hole low-energy high-resolution collimator at a speed of $12 \mathrm{~cm} / \mathrm{min}$. The images were acquired into a matrix size of $256 \times 1024$ with an energy window centered on the ${ }^{99 \mathrm{~m}} \mathrm{Tc} 140 \mathrm{keV}$ photopeak ( $\left.\pm 15 \%\right)$.

One-bed SPECT was acquired for the region of concern as detected on PBS. SPECT settings were $128 x$ 128 matrix over $360^{\circ}$ circular arc using 64 stops and 25 seconds per stop. Immediately after SPECT acquisition, low-dose CT images were obtained using a tube current of $80 \mathrm{~mA}$ and a voltage of $130 \mathrm{kV}$. The average radiation dose from low dose CT was $4 \mathrm{mGy} \pm 2.5$. The total SPECT/CT acquisition time was approximately $20 \mathrm{~min}$. CT images were reconstructed using a high-resolution reconstruction algorithm (B80 kernel) with 1-mm slice thickness.

Attenuation- and scatter-corrected SPECT images were reconstructed using the manufacturer's iterative protocol (Flash 3D) with 4 iterations, 4 subsets, and $8 \mathrm{~mm}$ Gaussian filter. Subsequently, tomographic slices were generated and displayed as transaxial, coronal, and sagittal slices. SPECT emission images were co-registered and fused with CT images and visually inspected for any misregistration.

\section{MRI imaging}

MR studies were conducted using a 1.5 Tesla MR machine (Magnetom Avanto, Siemens Medical Solutions, Erlangen, Germany) using an external coil array with subjects examined in the supine position. The different acquired planes and their parameters are summarized in Table 1. 
Table 1

Parameters of the employed MR sequences

\begin{tabular}{|c|c|c|c|c|c|}
\hline & $\begin{array}{l}\mathrm{TR} \\
\text { (msec) }\end{array}$ & $\begin{array}{l}\text { TE } \\
\text { (msec) }\end{array}$ & $\begin{array}{l}\text { FOV } \\
(\mathrm{mm})\end{array}$ & $\begin{array}{l}\text { FOV } \\
\text { Phase (\%) }\end{array}$ & $\begin{array}{l}\text { Slice } \\
\text { Thickness (mm) }\end{array}$ \\
\hline \multicolumn{6}{|l|}{ Spinal imaging } \\
\hline Sagittal T2WI & 3500 & 89 & 350 & 100 & 4.0 \\
\hline Sagittal T1WI & 590 & 11 & 280 & 100 & 4.0 \\
\hline Sagittal STIR & 3500 & 30 & 338 & 100 & 4.0 \\
\hline Coronal STIR & 3800 & 61 & 380 & 100 & 5.0 \\
\hline Axial T2WI & 2690 & 93 & 400 & 89 & 5.0 \\
\hline DWI & 5512 & 80 & 370 & 85 & 5.0 \\
\hline \multicolumn{6}{|l|}{ Pelvic imaging } \\
\hline Coronal STIR & 3500 & 61 & 380 & 100 & 5.0 \\
\hline Coronal T2WI & 3500 & 81 & 380 & 100 & 5.0 \\
\hline Sagittal T2WI & 3500 & 30 & 380 & 100 & 5.0 \\
\hline Axial T1WI & 500 & 11 & 420 & 75 & 4.0 \\
\hline DWI & 5800 & 83 & 440 & 81.3 & 4.5 \\
\hline \multicolumn{6}{|c|}{ Appendicular skeleton } \\
\hline Coronal STIR & 3500 & 40 & 130 & 100 & 3.0 \\
\hline Coronal T1WI & 523 & 18 & 120 & 100 & 3.0 \\
\hline Sagittal T2WI & 4000 & 78 & 120 & 100 & 3.0 \\
\hline Axial T2WI & 2690 & 93 & 400 & 92 & 3.0 \\
\hline Axial DWI & 5800 & 105 & 300 & 100 & 3.0 \\
\hline
\end{tabular}

Diffusion-weighted spin echo-planar images were covering the lumbar spine and pelvis mainly in axial plane and b values of 50,400 , and $800 \mathrm{sec} / \mathrm{mm}^{2}$ with SPAIR (Spectral Adiabatic Inversion Recovery) fat saturation. Using a Raw filter, anterior to posterior phase encoding [1]. Isotropic (ADC) maps generated with the vendor software employing all used b values.

\section{Image interpretation and follow-up}


Images from SPECT/CT were reviewed by two nuclear medicine physicians (R1; 5-year experience and R2;12-year experience). Areas with increased or focal uptake that does not conform with the normal distribution, without clear CT evidence of benign underlying etiology were considered malignant. Bone destruction on CT was also indicative of malignancy. All MRI images, including DWI, were reviewed by two radiologists (20-year experience and 19-year experience). Malignant lesions were defined on MRI as lesions that had low signal intensity on T1WI, iso- or high-signal intensity on $\mathrm{T} 2 \mathrm{WI}$, restricted diffusion, and high signal intensity on STIR sequence. Readers were aware of the patient's age and primary tumor type but were blinded to other clinical/imaging data, including the images from PBS. Accordingly, each lesion was evaluated according to a 5-point scale: score 1 = definitely benign, score 2 = probably benign, score 3 = equivocal, score 4 = probably malignant and score 5 = definitely malignant.

The true status of the lesion(s) was formulated as positive or negative based on subsequent clinical/imaging follow-up within six months. On imaging studies, size increase or changes in the character (osteolytic to sclerotic) were considered positive for malignancy. Stationary lesions without therapy were considered benign. In the presence of interval treatment, lesions that showed a response/stable course were considered malignant.

During analysis, lesions with scores 1 or 2 were considered benign, while those scored $3-5$ were considered malignant. Equivocal findings (score 3) were considered to the malignant side. The reason for that 'pessimistic' classification is that a report with equivocal findings would commonly necessitate further intervention (e.g., more imaging and closer follow-up).

\section{Statistical analysis}

Kappa test was used for assessing the degree of agreement between the readers. True-positive (TP), truenegative (TN), false-positive (FP), and false-negative (FN) readings were identified based on subsequent follow-up. Diagnostic performance parameters were calculated in the form of sensitivity, specificity, accuracy, positive predictive value (PPV), and negative predictive value (NPV). To limit the number of comparisons presented here, we compared only the results from the more experienced reader for each modality. The non-parametric McNemar's test was used to evaluate the statistical significance of the differences in sensitivity and specificity. The area under the curve (AUC) from receiver operating characteristic (ROC) analysis was used to demonstrate the overall accuracy of each modality using the 5points score. One radiologist measured the mean ADC value, and ROC analysis with 1000-sample bootstrapping of the Youden index was used to elicit the predictive accuracy of different mean ADC cutoff values in differentiating benign from malignant lesions.

Data were summarized as percentages, mean, and standard deviation. Confidence intervals were reported where applicable. In all analyses, P-value $<0.05$ (two-tailed) was considered statistically significant. The analysis was performed using SPSS v.21 (IBM Corp, Armonk, New York) and MedCalc (MedCalc Software, Mariakerke, Belgium).

\section{Results}




\section{Patients}

A total of 48 eligible patients were initially enrolled in this study. One patient was excluded due to lack of follow-up data. The other was excluded because the detected lesion on PBS was proved to be extraosseous on SEPCT/C; accordingly, did not perform MR-DWI. A valid cohort of 46 patients was available for analysis (32 females, 14 males), with a median age of 52 years (range: 10-77 years). The median interval between SPECT/CT and MRI studies was one week. Forty-four patients (92\%) had their MRI study performed within one month from SPECT/CT.

All the follow-up methods/procedures were performed at the discretion of the referring physician. The final diagnosis of bone lesions was obtained from subsequent imaging follow-up after a mean duration of $5.3 \pm 2.8$ months in 20 patients (18 with repeated bone scanning and SPECT/CT, 1 with MRI, and 1 with $\mathrm{X}$-ray,). Bone biopsy was performed in one patient only. The clinical course of the patient, as evaluated by the treating physician, after a mean follow-up duration of $5.0 \pm 1.6$ months, was considered the reference standard in the remaining 25 patients. Of these 25 patients, both bone SPECT/CT and MR-DWI were concordantly reported negative in 14 and positive in 5 patients. Thirty-one patients had no interval therapy between their initial scanning and follow-up, 12 had systemic therapy (hormonal or chemotherapy), and 3 had radiotherapy.

According to the final follow-up, the reference status of the 46 patients was classified as positive for osseous metastasis in 18 patients (39\%), while the remaining 28 patients $(61 \%)$ were considered diseasefree.

\section{Agreements within modalities}

Both radiologists perfectly agreed on describing the pattern of lesions in T2WI and STIR, and also on the presence or absence of soft tissue component $(k=1.0)$. In one patient only, there was no agreement on describing T1WI ( $\mathrm{k}=0.97 ; 95 \% \mathrm{Cl}$ : 0.9-1.0) or the presence of restriction on DWI ( $\mathrm{k}=0.95 ; 95 \% \mathrm{Cl}$ : 0.87-1.0). There was an identical positive/negative categorization between MRI readers $(k=1.0)$.

SPECT/CT demonstrated moderate agreement between raters $(k=0.6 ; 95 \% \mathrm{Cl}: 0.37-0.83 ; p<0.001)$. Raters agreed on classifying 22 patients as negative for metastasis and 15 as positive for metastases. Discordant categorization was seen in 9 patients $(20 \%)$.

\section{Diagnostic performance of SPECT/CT and MR-DWI}

Diagnostic performance indices are given in Table 2. Overall, SPECT/CT performed similar to MR-DWI with AUC of 0.77 (95\% Cl: $0.62-88)$ and 0.71 (95\% Cl: $0.55-0.83)$; respectively (Fig. 1). The difference between the two curves was not significant $(p=0.6)$. When the diffusion pattern is considered separately, restricted diffusion resulted in slightly lower sensitivity with comparable specificity when compared to the overall impression from all MRI sequences, including DWI. Lesions without restricted diffusion were mostly benign (20/26); however, only $8 / 19$ lesions that showed restricted diffusion were malignant ( $p=$ 
0.03). In one patient, no definite lesion was seen on MRI, and a separate evaluation of each of the MRI sequences, including DWI, was not possible. However, the study overall was considered negative.

Table 2

Diagnostic performance indices for the SPECT/CT and MR-DWI readers

\begin{tabular}{|c|c|c|c|c|}
\hline \multirow[t]{2}{*}{ Result } & \multicolumn{2}{|l|}{ SPECT/CT } & \multicolumn{2}{|l|}{ MR-DWI } \\
\hline & R1 & $\mathrm{R} 2$ & R1 & $\mathrm{R} 2$ \\
\hline FN & 4 & 6 & 5 & 5 \\
\hline TP & 14 & 12 & 13 & 13 \\
\hline TN & 21 & 22 & 23 & 23 \\
\hline FP & 7 & 6 & 5 & 5 \\
\hline Sensitivity & $78(52-94)$ & $67(41-87)$ & $72(47-90)$ & $72(47-90)$ \\
\hline Specificity & $75(55-89)$ & $79(59-92)$ & $82(63-94)$ & $82(63-94)$ \\
\hline AUC\% & $78(64-92)$ & $79(65-93)$ & $72(56-88)$ & $73(57-89)$ \\
\hline PPV & $67(43-85)$ & $67(41-87)$ & $72(47-90)$ & $72(47-90)$ \\
\hline NPV & $84(64-95)$ & $79(59-92)$ & $82(63-94)$ & $82(63-94)$ \\
\hline LR+ & $3.1(1.6-6.2)$ & $3.1(1.4-6.8)$ & $4.0(1.7-9.4)$ & $4.0(1.7-9.4)$ \\
\hline LR- & $0.3(0.1-0.7)$ & $0.4(0.2-0.8)$ & $0.3(0.2-0.7)$ & $0.3(0.2-0.7)$ \\
\hline \multicolumn{5}{|c|}{ The numbers in parenthesis are the $95 \%$ confidence interval } \\
\hline \multicolumn{5}{|c|}{$\begin{array}{l}\text { MR-DWI = magnetic resonance with diffusion weighted imaging, } R 1=\text { reader } 1, R 2=\text { Reader } 2, A U C= \\
\text { area under the curve for the } 5 \text {-point score, } F N=\text { false negative, TP }=\text { true positive, } T N=\text { true negative, } \\
F P=\text { false positive, } P P V=\text { positive predictive value, NPV }=\text { negative predictive value, } L R+=\text { positive } \\
\text { likelihood ratio, LR-=negative likelihood ratio }\end{array}$} \\
\hline
\end{tabular}

Both SPECT/CT (R2) and MR-DWI (R1) agreed on the classification of 8 TP and 18 TN lesions. SPECT/CT correctly categorized disease in 4/5 FN lesions seen on MR-DWI (Fig. 2), while MR-DWI correctly diagnosed malignancy in 5/6 FN results from SPECT/CT. Similarly, SPECT/CT correctly excluded disease in 4/5 FP lesions by MR-DWI, while MR-DWI correctly re-categorized 5/6 FP readings from SPECT/CT as TN. There was no difference in sensitivity or specificity.

The performance of the two modalities was affected by the primary tumor site, lesion site, and CT pattern (Table 3). The overall accuracy was slightly lower for patients with breast cancer, vertebral metastasis, or non-sclerotic lesions. 
Table 3

Area under the curve (AUC) of the SPECT/CT and MR-DWI readings according to the primary tumor type, lesion site and lesion pattern on CT

\begin{tabular}{|c|c|c|c|c|c|}
\hline \multirow[t]{2}{*}{ Sub-group } & \multirow[t]{2}{*}{$\mathbf{N}$} & \multicolumn{2}{|c|}{ SPECT/CT } & \multicolumn{2}{|l|}{ MR-DWI } \\
\hline & & R1 & R2 & R1 & R2 \\
\hline \multicolumn{6}{|l|}{ Primary tumor } \\
\hline Non-breast & 21 & $\begin{array}{l}94 \% \\
(75-1.0)\end{array}$ & $\begin{array}{l}77 \% \\
(53-92)\end{array}$ & $\begin{array}{l}85 \% \\
(63-97)\end{array}$ & $\begin{array}{l}85 \% \\
(63-97)\end{array}$ \\
\hline Breast & 25 & $\begin{array}{l}63 \% \\
(42-81)\end{array}$ & $\begin{array}{l}75 \% \\
(53-90)\end{array}$ & $\begin{array}{l}59 \% \\
(37-78)\end{array}$ & $\begin{array}{l}59 \% \\
(38-78)\end{array}$ \\
\hline \multicolumn{6}{|l|}{ Lesion site } \\
\hline Appendicular & 16 & $\begin{array}{l}83 \% \\
(56-97)\end{array}$ & $\begin{array}{l}88 \% \\
(62-98)\end{array}$ & $\begin{array}{l}92 \% \\
(68-1.0)\end{array}$ & $\begin{array}{l}92 \% \\
(68-1.0)\end{array}$ \\
\hline Axial & 30 & $\begin{array}{l}82 \% \\
(63-93)\end{array}$ & $\begin{array}{l}78 \% \\
(59-91)\end{array}$ & $\begin{array}{l}64 \% \\
(44-80)\end{array}$ & $\begin{array}{l}65 \% \\
(45-81)\end{array}$ \\
\hline \multicolumn{6}{|c|}{ Involved location } \\
\hline Spine & 20 & $\begin{array}{l}68 \% \\
(44-87)\end{array}$ & $\begin{array}{l}75 \% \\
(51-91)\end{array}$ & $\begin{array}{l}64 \% \\
(39-84)\end{array}$ & $\begin{array}{l}63 \% \\
(39-83)\end{array}$ \\
\hline Other sites & 26 & $\begin{array}{l}82 \% \\
(62-94)\end{array}$ & $\begin{array}{l}84 \% \\
(65-95)\end{array}$ & $\begin{array}{l}79 \% \\
(58-92)\end{array}$ & $\begin{array}{l}80 \% \\
(59-93)\end{array}$ \\
\hline \multicolumn{6}{|l|}{ CT pattern } \\
\hline Sclerotic & 22 & $\begin{array}{l}74 \% \\
(52-90)\end{array}$ & $\begin{array}{l}72 \% \\
(49-89)\end{array}$ & $\begin{array}{l}73 \% \\
(50-90)\end{array}$ & $\begin{array}{l}73 \% \\
(50-90)\end{array}$ \\
\hline Not sclerotic & 24 & $\begin{array}{l}68 \% \\
(44-87)\end{array}$ & $\begin{array}{l}75 \% \\
(51-91)\end{array}$ & $\begin{array}{l}64 \% \\
(39-84)\end{array}$ & $\begin{array}{l}63 \% \\
(39-83)\end{array}$ \\
\hline \multicolumn{6}{|c|}{ The numbers in parenthesis are the $95 \%$ confidence interval } \\
\hline MR-DWI = & & $c 0$ & 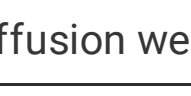 & бес иाта & \\
\hline
\end{tabular}

\section{Primary tumor site}

Breast cancer was seen in $54 \%$ of this cohort $(n=25)$. Other tumor sites included: urinary bladder cancer $(n=6)$, lymphoma $(n=2)$, and lung cancer $(n=2)$. Other primary tumors encountered were osteosarcoma, Ewing's sarcoma, medullary thyroid cancer, gall bladder carcinoma, renal cell carcinoma, spindle cell 
tumor, liver, prostate, endometrial, pancreatic and tongue cancers $(n=1$ each). Since breast cancer represented more than half of our cohort, a sub-analysis of this group might be relevant. Osseous metastasis was seen in 11/25 patients with breast cancer and $6 / 21$ patients with non-breast cancer. Overall accuracy (Table 3 ) and all other diagnostic indices were slightly better for both modalities in the non-breast cancer group.

Vertebral lesions were encountered slightly more frequently among breast cancer group; however, the overall distribution of appendicular and axial lesions did not vary significantly according to primary tumor type or lesion nature on CT (Table 4).

Table 4

The nature of lesions detected on CT part of the SPECT/CT, classified according to the primary tumor, involved site and final status

\begin{tabular}{|c|c|c|c|c|c|c|}
\hline \multirow[t]{2}{*}{ Lesion nature } & \multirow[t]{2}{*}{ Primary tumor } & \multirow[t]{2}{*}{ Site involved } & \multicolumn{4}{|c|}{ Lesion Nature on CT } \\
\hline & & & Lytic & Sclerotic & Mixed & No CT Changes \\
\hline \multirow[t]{4}{*}{ Benign } & \multirow[t]{2}{*}{ Non-breast } & Spine & 0 & 1 & 1 & 3 \\
\hline & & Other sites & 0 & 6 & 1 & 3 \\
\hline & \multirow[t]{2}{*}{ Breast cancer } & Spine & 0 & 3 & 1 & 3 \\
\hline & & Other sites & 2 & 3 & 0 & 1 \\
\hline \multirow[t]{4}{*}{ Malignant } & \multirow[t]{2}{*}{ Non-breast } & Spine & 0 & 3 & 0 & 0 \\
\hline & & Other sites & 1 & 0 & 1 & 1 \\
\hline & \multirow[t]{2}{*}{ Breast cancer } & Spine & 2 & 2 & 1 & 0 \\
\hline & & Other sites & 1 & 4 & 2 & 0 \\
\hline
\end{tabular}

\section{Site of skeletal lesion}

Most skeletal lesions that were located in the axial skeleton were benign (20/30) compared to 8/16 of appendicular lesions. The spine was the most common site for axial lesions (7/20 were malignant). Other malignant axial lesions sites included: skull (1/4), posterior chest wall $(0 / 3)$, sternum (1/2), and sacrum $(1 / 1)$.

MR-DWI was more accurate for lesions located in the appendicular skeleton compared to axial lesions (Table 3). The performance was comparable when the spine was considered against other axial and appendicular sites. On the other hand, SPECT/CT did not show significant variability in the overall accuracy according to the lesion site (Table 3).

\section{Nature of the lesions on CT}

The spectrum of CT findings seen in the lesions that were proved benign included vertebral wedging, hemangioma, fractures, degenerative changes, spondylodiscitis, arthritis, bone cyst, and bone island. No 
CT findings were encountered in 10 benign lesions and one malignant lesion, which was proved to be due to malignant marrow infiltration with no obvious cortical component.

Lesions were sclerotic $(n=22)$, lytic $(n=6)$ or mixed $(n=7)$. Six of the 11 false results on MRI were sclerotic on CT; 3 of these 6 lesions were located in the spine; 2 of them were secondary to vertebral wedging (1 FN, $1 \mathrm{FP})$, and one falsely interpreted as Schmorl's nodule.

\section{Analysis of different MRI sequences and mean ADC}

The pattern of qualitative MRI features associated with malignancy was mostly hypointense signal on T1WI (52\%), hyperintensity on STIR (65\%), and restricted diffusion (65\%).

$A D C$ value was not predictor of lesion status with AUC of $0.58(95 \% \mathrm{Cl}=0.42-0.72 ; p=0.4)$. The analysis identified a mean $A D C$ value of $\leq 0.8 \times 10^{-3} \mathrm{~mm}^{2} / \mathrm{s}$ as the most discriminative cut-off value. This point resulted in good specificity of $74 \%$, albeit with modest sensitivity of $50 \%$.

\section{Discussion}

Multimodality evaluation of equivocal osseous lesions can potentially overcome the limitations of each imaging modality and favorably impact lesion characterization. However, access to these imaging services may not be available at all geographic locations. Therefore, reaching a practical institutional algorithm to diagnose these lesions could save resources and improve outcomes.

In this study, we evaluated the diagnostic performance from SPECT/CT and MRI with diffusion-weight imaging (DWI) for characterizing the nature of solitary osseous lesions that were classified as indeterminate based on conventional PBS.

Two raters independently evaluated each SPECT/CT study. In contrast to the study of Sharma et al. [23], the overall performance of SPECT/CT reported in our study was lower and also the level of agreement. Their study focused on lung cancer patients only with a high prevalence of metastatic osseous disease (70\%) compared to $37 \%$ in our study. Disease prevalence could impact diagnostic performance indices [24]. Also, treating equivocal lesion as malignant (pessimistic analysis) could shift the values of sensitivity and specificity $[25,26]$.

Furthermore, the difference in years of experience in reading nuclear medicine studies in general and SPECT/CT, in particular, could lead to variable results between centers. On the other hand, the two radiologists demonstrated perfect agreement on scoring MR-DWI studies.

In this study, no significant difference could be seen between the two modalities for any of the diagnostic performance indices. To our knowledge, no prior studies directly compared MR-DWI and SPECT/CT in the proposed clinical setting where PBS detects solitary equivocal lesions. Previous reports, performed in a different clinical context, compared MR-DWI to PBS and documented comparable results between the two modalities [27]. One large study [28] compared whole-body scans using DWI and SPECT in addition to 
other modalities (conventional MRI and PET/CT) in 115 patients with lung cancer. The reported specificity and accuracy of DWI alone were lower than those of SPECT for 1025 sites ( $93.7 \% \mathrm{vs.} 95.0$ and $93.9 \%$ vs. $95 \%$, respectively). However, the addition of conventional WB MRI resulted in significantly higher specificity (96.1\%) and accuracy (96.1\%) over SPECT alone. In the same work, the addition of DWI improved overall accuracy when compared to either conventional MRI or DWI alone [28]. These results, though not directly comparable to our study, may suggest that isolated DWI may not be superior to SPECT. In a meta-analysis [18] which included data from 32 studies with 1507 patients, the pooled sensitivity, specificity, and AUC of MR-DWI were $0.95,0.92$ and 0.98 on a per-patient basis, and $0.91,0.94$, and 0.97 on a per lesion basis. There was no statistically significant difference found in the sensitivity and specificity of using DWI only and DWI combined with other morphological or functional imaging sequences for either analysis.

We did not perform an independent evaluation of DWI alone for discriminating benign versus malignant features. It has been recommended that DWI should be interpreted with other imaging sequences because benign lesions may result in false positives [29], and certain malignant lesions, such as sclerotic bone metastases, can lead to false-negative results [30, 31]. However, if restricted diffusion alone were used as a criterion for malignancy in our study, the sensitivity and specificity would be $63 \%(10 / 16)$ and $72 \%(20 / 28)$, respectively.

In the current work, $6 / 11$ false MRI results were sclerotic on CT part of the SPECT/CT, with half of them located in the spine (3/6). Common causes of false results on MRI include hematopoietic islands (e.g., secondary to use of hematopoietic growth factors), benign compression fractures (e.g., osteoporotic), lesions confined to the cortex only [32], and sclerotic lesions especially after therapy [33, 34]; as a response to treatment may result in edema, the return of marrow fat, fibrosis, and sclerosis [35]. It worth noting that nearly $25 \%$ of our study participants were receiving some sort of systemic therapy at the time of this study.

Previous studies reported significantly lower ADC values for malignant lesions. However, the ADC cut-off value for distinguishing between normal and malignant bone marrow was quite variable in prior works (examples were $0.5 \times 10^{-3} \mathrm{~mm}^{2} / \mathrm{s}$ [4], $0.6 \times 10^{-3} \mathrm{~mm}^{2} / \mathrm{s}$ [2], $0.7 \times 10^{-3} \mathrm{~mm}^{2} / \mathrm{s}$ [1], and $0.9710^{-3} \mathrm{~mm}^{2} / \mathrm{s}$ [3]). In our study, the mean ADC did not show a statistically significant association with the final status. However, our best discriminatory ADC cut-off value falls within the previously reported range $[15,16,36$, 37]. Furthermore, the quantitative assessment of $A D C$ solely via its mean value, as performed in the current report, might not yield a proper discriminating criterion between benign and malignant lesions, and obtaining multiple quantitative metrics is encouraged.

The discrepancy of ADC values and different cut-off values is likely attributed to the choice of b-value, methods of fat suppression, and the bone marrow cellular density and matrix composition of the bony lesions. For example, osteolytic lesions are better detected on DWI due to a higher content of cellularity and water within the lesions $[1,2,6,7]$. In our study, the employed b-factors were $50,400,800 \mathrm{~s} / \mathrm{mm}^{2}$, and the generated ADC values were quite different as we did not employ b0 as in the previous work by 
Messiou et al. [2]. Also, the age of our study population had a wide range (10-70 years), which caused variations in bone marrow constituents and distribution (e.g., red vs. yellow bone marrow). The yellow bone marrow is characterized by low cell density, an abundance of fat cells, reduced water protons, and hydrophobic nature of fat in addition to lower bone perfusion compared to the red bone marrow [6]. However, there are overlapping morphological signal characteristics between yellow bone marrow and neoplastic tissue [4]. Tumor cells infiltrating bone marrow displace fat cells, thus increasing cellular and water proton density, and augmenting blood perfusion; accordingly, malignant yellow bone marrow lesions show high signal intensity on DWI, but higher ADC values than normal yellow bone marrow. In contrast, soft tissue malignant lesions show high signal intensity on DWI and lower ADC values than the corresponding normal tissue [6].

Our current results concurred with the conclusions from many prior works, which emphasized the multiple constraints for the application of DWI in the evaluation of oncological patients, including, the high cellularity lesions such as sclerotic lesions, red/yellow bone marrow matrix, malignant lesions with less evident on the normal background and treatment with bone marrow stimulating factors as Granulocyte colony-stimulating factor (G-CSF) $[2,6,7]$. The currently-reported unsatisfactory performance of DWI supports the conclusion of Grankvist et al.[7] who concluded that DWI cannot replace T1 and STIR sequences but should be added to improve diagnostic efficacy

Our study has multiple limitations, including a small sample size, which reduced the power of our conclusions and made sub-group analysis more challenging. Because there were no prior reports directly comparing the two modalities in this specific clinical setting, the results from our exploratory study could be used to power and inform future studies. Another disadvantage is the inclusion of patients was based on bone scan findings; we understand that bone scan may lack adequate sensitivity for detecting small early marrow lesions and those with pure lytic nature; however, we aimed to reproduce the commonly utilized clinical scenario, in which bone scintigraphy is the first performed screening modality for detecting osseous metastasis, then cross-sectional imaging is added for equivocal or indeterminate findings.

Another limitation is that clinical MRI protocols may vary by the region scanned. Also, we used the pattern from all MRI sequences, including DWI, to reach a diagnostic score, hence, isolating the impact of adding DWI was not possible with the current study design. Furthermore, MRI has more advantages in evaluating soft tissues even in the same procedures, which was not evaluated in this study.

A common disadvantage of many imaging studies that discuss the problem of indeterminate osseous lesions is the absence of histopathological validation in the majority of patients. Bone biopsy for the sole purpose of research could be both technically challenging and ethically unjustifiable. Further larger prospective studies are needed to address the current limitations and confirm our findings.

In conclusion, we performed a prospective comparison between MR-DWI and SPECT/CT for characterizing solitary indeterminate osseous lesions seen on standard planar bone scans. MR-DWI results were more reprodicuble among readers. However, no significant difference in diagnostic 
performance was depicted between the two modalities. The overall accuracy of MRI varied according to the lesion site, the pattern on CT, and the primary tumor type. Such differences were less obvious with SPECT/CT.

\section{Abbreviations}

SPECT/CT

single photon emission computed tomography/computed tomography

MR-DWI

magnetic resonance with diffusion weighted imaging

STIR

Short tau inversion recovery

ADC

apparaent diffusion co-efficient

PBS

planar bone scintigraphy

$\mathrm{Cl}$

confidence interval

AUC

area under the curve

ROC

receiver operating characteristics

FN

false negative

TP

true positive

TN

true negative

FP

false positive

PPV

positive predictive value

NPV

negative predictive value

LR+

positive likelihood ratio

LR-

negative likelihood ratio.

\section{Declarations}


Acknowledgements

None

\section{Authors' contributions}

Conceptualization, MK, YGA and HA; Methodology, MK, YGA, GSS, and HA; Formal analysis, and investigation, MK, YGA, GSS and HA; Resources, YGA, AAK; Data curation, MK; Writing-original draft preparation, MK, and YGA; Writing-review and editing, MK, YGA, AAK, GSS, and HA; Visualization, YGA, and HA; Supervision, AAK, YGA, and HA; Project administration, YGA, and HA. All authors read and approved the final manuscript.

\section{Funding}

None

\section{Availability of data and materials}

The authors confirm that the data supporting the findings of this study are available within the article.

\section{Ethical approval and consent to participate}

All procedures performed in studies involving human participants were in accordance with the ethical standards of the institutional research committee and with the 1964 Declaration of Helsinki and its later amendments or comparable ethical standards. This article does not contain any studies with animals performed by any of the authors.

\section{Consent for publication}

Consent for publication was obtained from all individual participants included in the study.

\section{Competing interests}

The authors declare no conflict of interest.

\section{Author details}

Maha Khalil, MD, ${ }^{1}$, Yasser G. Abdelhafez, MD, PhD ${ }^{1,2}$, Ahmed A. Kandeel, MD, PhD ${ }^{3}$ Gehan S Seifeldein, $\mathrm{MD}, \mathrm{PhD}^{4}$, Haisam Atta, MD, $\mathrm{PhD}^{5}$

6. Nuclear Medicine Unit, South Egypt Cancer Institute, Assiut University, Egypt.

7. Department of Radiology, University of California Davis, Sacramento, California, USA

8. Oncology and Nuclear Medicine Department (NEMROCK), Kasr-El-Ainy Hospital, Cairo University, Egypt. 
9. Department of diagnostic Radiology, Assiut University Hospitals, Faculty of Medicine, Assiut University, Egypt

10. Radiology Department, South Egypt Cancer Institute, Assiut University, Egypt.

\section{References}

1. Roodman GD. Mechanisms of bone metastasis. N Engl J Med 2004; 350 (16):1655-1664.

2. Brenner Al, Koshy J, Morey J, Lin C, DiPoce J. The bone scan. Semin Nucl Med 2012; 42 (1):11-26.

3. Gralow JR, Biermann JS, Farooki A, Fornier MN, Gagel RF, Kumar R, et al. NCCN Task Force Report: Bone Health In Cancer Care. J Natl Compr Canc Netw 2013; 11 Suppl 3:S1-50; quiz S51.

4. Petersen LJ, Strandberg J, Stenholt L, Johansen MB, Zacho HD. Reporting and Handling of Indeterminate Bone Scan Results in the Staging of Prostate Cancer: A Systematic Review. Diagnostics (Basel) 2018; 8 (1).

5. Gayed IW, Kim EE, Awad J, Joseph U, Wan D, John S. The value of fused SPECT/CT in the evaluation of solitary skull lesion. Clin Nucl Med 2011; 36 (7):538-541.

6. Iqbal B, Currie GM, Wheat JM, Raza H, Kiat H. The incremental value of SPECT/CT in characterizing solitary spine lesions. Journal of nuclear medicine technology 2011; 39 (3):201-207.

7. Mahaletchumy T, AbAziz A. Incremental Value of Single-photon Emission Computed Tomographycomputed Tomography for Characterization of Skeletal Lesions in Breast Cancer Patients. World J Nucl Med 2017; 16 (4):303-310.

8. lagaru A, Young P, Mittra E, Dick DW, Herfkens R, Gambhir SS. Pilot prospective evaluation of 99mTcMDP scintigraphy, 18F NaF PET/CT, 18F FDG PET/CT and whole-body MRI for detection of skeletal metastases. Clin Nucl Med 2013; 38 (7):e290-296.

9. Zhang Y, Shi H, Gu Y, Xiu Y, Li B, Zhu W, et al. Differential diagnostic value of single-photon emission computed tomography/spiral computed tomography with Tc-99m-methylene diphosphonate in patients with spinal lesions. Nucl Med Commun 2011; 32 (12):1194-1200.

10. Helyar V, Mohan HK, Barwick T, Livieratos L, Gnanasegaran G, Clarke SE, et al. The added value of multislice SPECT/CT in patients with equivocal bony metastasis from carcinoma of the prostate. Eur J Nucl Med Mol Imaging 2010; 37 (4):706-713.

11. Zhao Z, Li L, Li F, Zhao L. Single photon emission computed tomography/spiral computed tomography fusion imaging for the diagnosis of bone metastasis in patients with known cancer. Skeletal radiology 2010; 39 (2):147-153.

12. Mundy GR. Metastasis to bone: causes, consequences and therapeutic opportunities. Nat Rev Cancer 2002; 2 (8):584-593.

13. Frank JA, Ling A, Patronas NJ, Carrasquillo JA, Horvath K, Hickey AM, et al. Detection of malignant bone tumors: MR imaging vs scintigraphy. AJR Am J Roentgenol 1990; 155 (5):1043-1048. 
14. Yang H-L, Liu T, Wang X-M, Xu Y, Deng S-M. Diagnosis of bone metastases: a meta-analysis comparing 18FDG PET, CT, MRI and bone scintigraphy. European radiology 2011; 21 (12):2604-2617.

15. Padhani AR, van Ree K, Collins DJ, D'Sa S, Makris A. Assessing the relation between bone marrow signal intensity and apparent diffusion coefficient in diffusion-weighted MRI. Am J Roentgenol 2013; 200 (1):163-170.

16. Sung JK, Jee W-H, Jung J-Y, Choi M, Lee S-Y, Kim Y-H, et al. Differentiation of acute osteoporotic and malignant compression fractures of the spine: use of additive qualitative and quantitative axial diffusion-weighted MR imaging to conventional MR imaging at 3.0 T. Radiology 2014; 271 (2):488498.

17. Khoo MM, Tyler PA, Saifuddin A, Padhani AR. Diffusion-weighted imaging (DWI) in musculoskeletal MRI: a critical review. Skeletal radiology 2011; 40 (6):665-681.

18. Liu L-P, Cui L-B, Zhang X-X, Cao J, Chang N, Tang X, et al. Diagnostic Performance of Diffusionweighted Magnetic Resonance Imaging in Bone Malignancy: Evidence From a Meta-Analysis. Medicine 2015; 94 (45):e1998.

19. Nemeth A, Henson J, Mullins M, Gonzalez R, Schaefer P. Improved detection of skull metastasis with diffusion-weighted MR imaging. American journal of neuroradiology 2007; 28 (6):1088-1092.

20. Messiou C, Collins D, Morgan V. Optimising diffusion weighted MRI for imaging metastatic and myeloma bone disease and assessing reproducibility. European radiology 2011; 21 (8):1713-1718.

21. Mosavi F, Johansson S, Sandberg DT, Turesson I, Sorensen J, Ahlstrom H. Whole-body diffusionweighted MRI compared with (18)F-NaF PET/CT for detection of bone metastases in patients with high-risk prostate carcinoma. AJR Am J Roentgenol 2012; 199 (5):1114-1120.

22. Webster E, Alpert N, Brownell G. Radiation doses in pediatric nuclear medicine and diagnostic x-ray procedures. In: James AE, Wagner HN, Cooke RE, eds. Pediatric nuclear medicine. Philadelphia,: Saunders, 1974: pp. xvi, 544 p.

23. Sharma P, Kumar R, Singh H, Bal C, Julka PK, Thulkar S, et al. Indeterminate lesions on planar bone scintigraphy in lung cancer patients: SPECT, CT or SPECT-CT? Skeletal radiology 2012; 41 (7):843850.

24. Whiting P, Rutjes AW, Reitsma JB, Glas AS, Bossuyt PM, Kleijnen J. Sources of variation and bias in studies of diagnostic accuracy: a systematic review. Ann Intern Med 2004; 140 (3):189-202.

25. Rager O, Nkoulou R, Exquis N, Garibotto V, Tabouret-Viaud C, Zaidi H, et al. Whole-Body SPECT/CT versus Planar Bone Scan with Targeted SPECT/CT for Metastatic Workup. Biomed Res Int 2017; 2017:7039406.

26. Fleury V, Ferrer L, Colombie M, Rusu D, Le Thiec M, Kraeber-Bodere F, et al. Advantages of systematic trunk SPECT/CT to planar bone scan (PBS) in more than 300 patients with breast or prostate cancer. Oncotarget 2018; 9 (60):31744-31752.

27. Nakanishi K, Kobayashi M, Nakaguchi K, Kyakuno M, Hashimoto N, Onishi H, et al. Whole-body MRI for Detecting Metastatic Bone Tumor: Diagnostic Value of Diffusion-weighted Images. Magnetic Resonance in Medical Sciences 2007; 6 (3):147-155. 
28. Takenaka D, Ohno Y, Matsumoto K, Aoyama N, Onishi Y, Koyama H, et al. Detection of bone metastases in non-small cell lung cancer patients: comparison of whole-body diffusion-weighted imaging (DWI), whole-body MR imaging without and with DWI, whole-body FDG-PET/CT, and bone scintigraphy. J Magn Reson Imaging 2009; 30 (2):298-308.

29. Grankvist J, Fisker R, lyer V, Frund ET, Simonsen C, Christensen T, et al. MRI and PET/CT of patients with bone metastases from breast carcinoma. Eur J Radiol 2012; 81 (1):e13-18.

30. Koh D-M, Takahara T, Imai Y, Collins DJ. Practical Aspects of Assessing Tumors Using Clinical Diffusion-weighted Imaging in the Body. Magnetic Resonance in Medical Sciences 2007; 6 (4):211224.

31. Lecouvet FE, El Mouedden J, Collette L, Coche E, Danse E, Jamar F, et al. Can whole-body magnetic resonance imaging with diffusion-weighted imaging replace Tc $99 \mathrm{~m}$ bone scanning and computed tomography for single-step detection of metastases in patients with high-risk prostate cancer? Eur Urol 2012; 62 (1):68-75.

32. Taoka T, Mayr NA, Lee HJ, Yuh WT, Simonson TM, Rezai K, et al. Factors influencing visualization of vertebral metastases on MR imaging versus bone scintigraphy. AJR Am J Roentgenol 2001; 176 (6):1525-1530.

33. Shah LM, Salzman KL. Imaging of spinal metastatic disease. Int J Surg Oncol 2011; 2011:769753.

34. Subhawong TK, Jacobs MA, Fayad LM. Insights into quantitative diffusion-weighted MRI for musculoskeletal tumor imaging. AJR Am J Roentgenol 2014; 203 (3):560-572.

35. Lecouvet FE, Talbot JN, Messiou C, Bourguet P, Liu Y, De Souza NM. Monitoring the response of bone metastases to treatment with Magnetic Resonance Imaging and nuclear medicine techniques: A review and position statement by the European Organisation for Research and Treatment of Cancer imaging group. European Journal of Cancer 2014; 50 (15):2519-2531.

36. Neubauer H, Evangelista L, Hassold N, Winkler B, Schlegel PG, Köstler $H$, et al. Diffusion-weighted MRI for detection and differentiation of musculoskeletal tumorous and tumor-like lesions in pediatric patients. World Journal of Pediatrics 2012; 8 (4):342-349.

37. Wang T, Wu X, Cui Y, Chu C, Ren G, Li W. Role of apparent diffusion coefficients with diffusionweighted magnetic resonance imaging in differentiating between benign and malignant bone tumors. World journal of surgical oncology 2014; 12 (1):1.

\section{Figures}




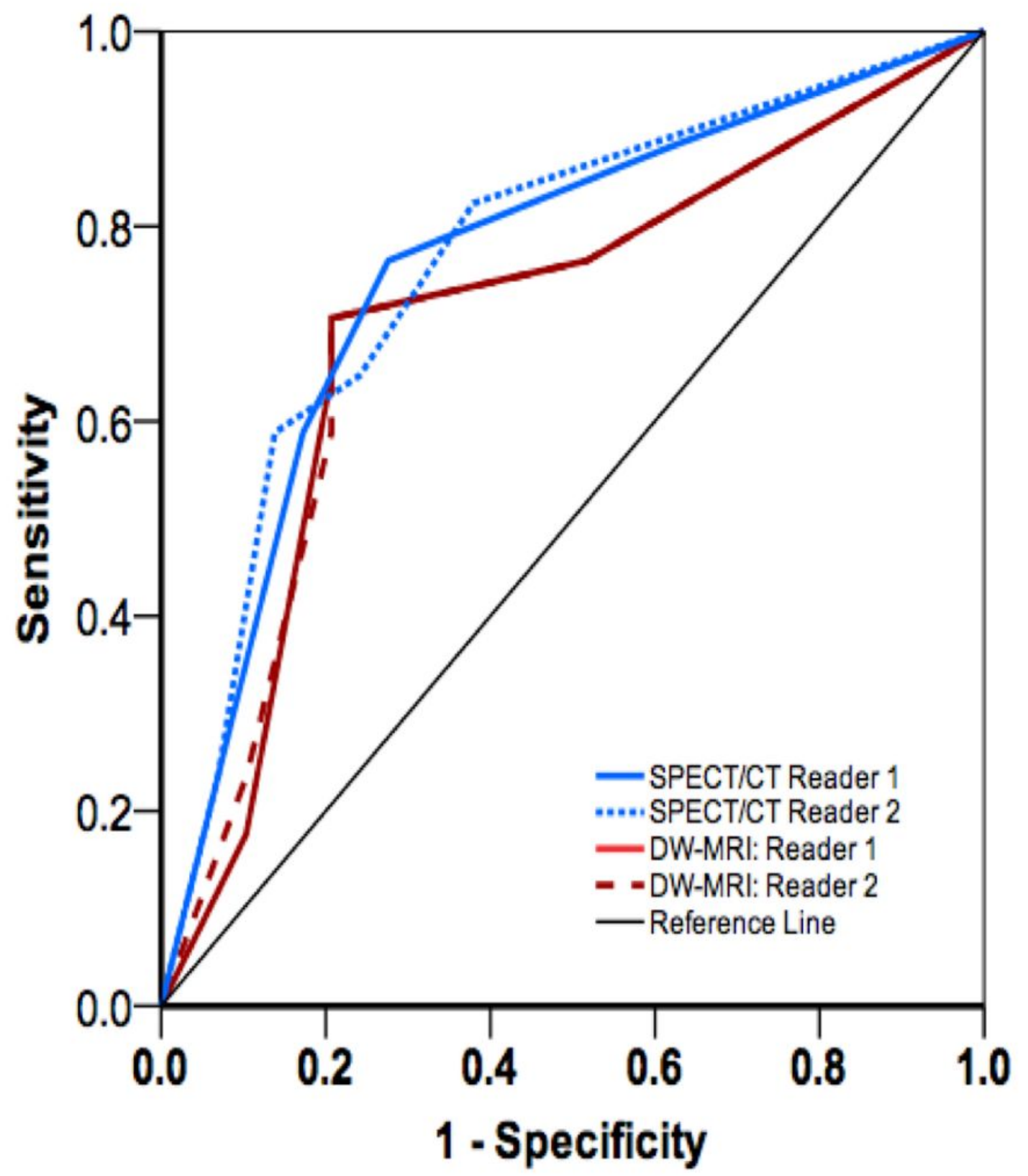

Diagonal segments are produced by ties.

Figure 1

Area under receiver operating characteristic curves for the 5-point score readings from SPECT/CT and DW-MRI 


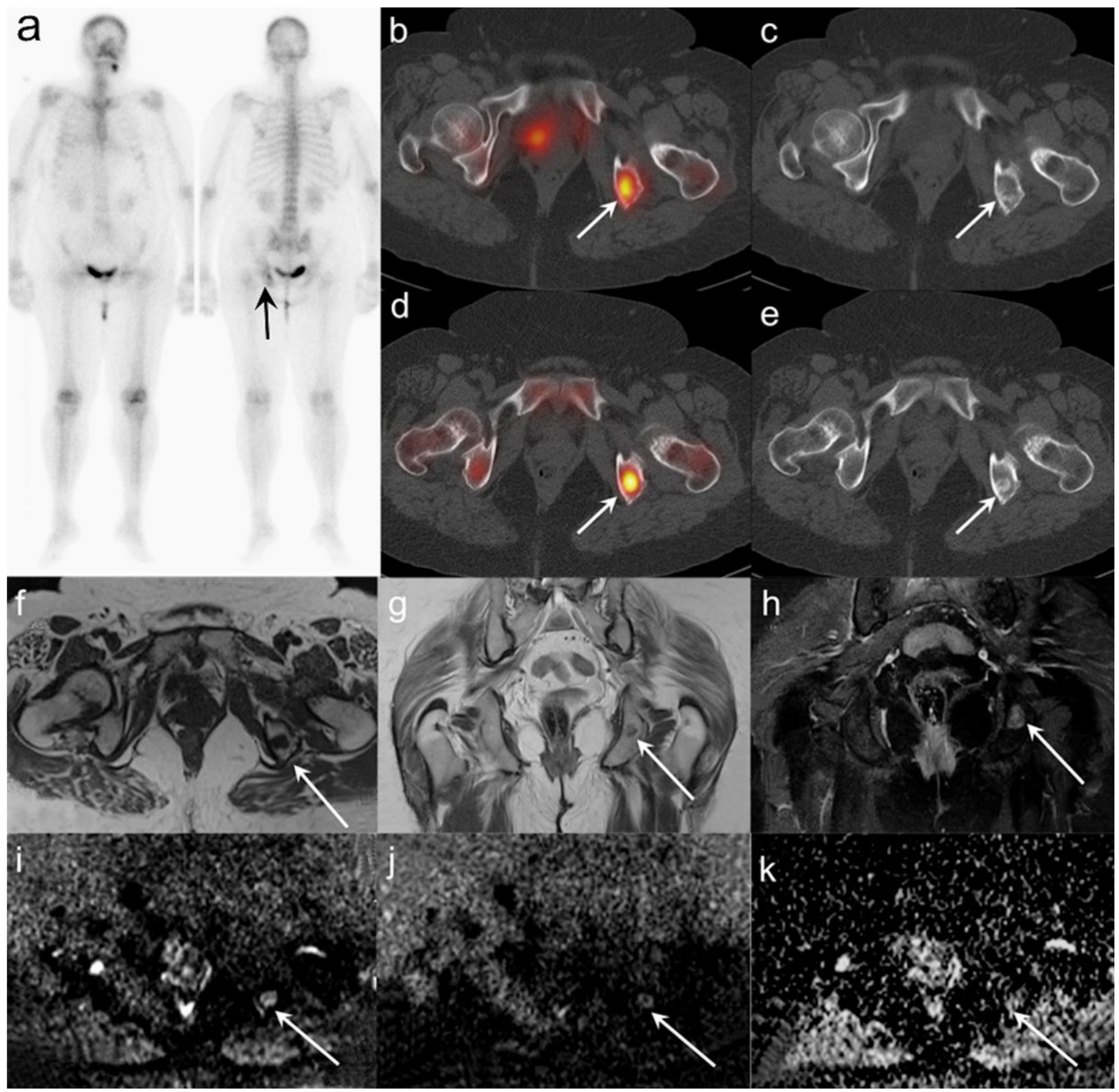

Figure 2

An example of true positive lesion in SPECT/CT with false negative DW-MRI findings. A 55-year-old woman with right breast cancer status post modified radical mastectomy and adjuvant chemo- and radiotherapy. Anterior and posterior whole-body planar bone scan a shows faint tracer uptake at the left ischium (black arrow on posterior view). Fused SPECT/CT images $b$ demonstrating focal tracer uptake (white arrow) that corresponds to a subtle area of sclerosis (white arrow) in low-dose CT c. Conventional MRI images $\mathrm{f}-\mathrm{h}$ demonstrate heterogeneous signal intensity (white arrow), exhibiting hypointense signal in axial T1 WI f, faint hypointense in coronal T2 WI g, and hyperintense signal in coronal STIR WI h. 
Facilitated diffusion is noted in DWI i-k (b factor 50 in i \& 800 in j) with relatively high signal in ADC map k. Mean ADC value is $1.2 \times 10-3 \mathrm{~mm} 2 / \mathrm{sec}$. Follow-up SPECT/CT images $d-e, 3$ months later with no interval therapy, demonstrate progression of the previously noted focal activity in the left ischium $d$, corresponding to a well-seen sclerotic lesion in low-dose CT images e. 